\title{
Design of Ultrasonic Ranging System for Marking Vehicle
}

\author{
Yu Haiming ${ }^{1, a}$, Kang Rui ${ }^{2, b}$, Li Shi ${ }^{3, c}$, Chen Kunjie ${ }^{4, d}$ \\ 1,2,3,4No.40,Dianjiangtai Road, Pukou District, Nanjing City, Jiangsu Province, PRC \\ ayuhaiming@njau.edu.cn, ${ }^{b} 504662849 @ q q . c o m,{ }^{c}$ shili@niau.edu.cn, ${ }^{d}$ kunjiechen@njau.edu.cn
}

\section{Keywords: Ultrasonic wave; Marking; Vehicle}

\begin{abstract}
In this paper, the authors designed ultrasonic ranging system for marking vehicle. This system consists of an ultrasonic module, DSP, temperature compensating module, LCD and keyboard. LM1812 was adopted to transmit and receive ultrasonic wave information. The digital signal processor TMS320LF2407 was used to control the ultrasonic system, select and process data. The distance between road-lineation vehicle and the reference objective was calculated based on reflecting time of ultrasonic wave and thus the accuracy position of the vehicle was determined. The simulation test showed that the measuring range of ultrasonic ranging system was $60 \sim 700 \mathrm{~cm}$. The measuring errors of the ultrasonic ranging system were less in the range of $100 \sim 500 \mathrm{~cm}$.
\end{abstract}

\section{Introduction}

Road marking, one important index for checking and evaluating highway engineering quality, plays an essential role in controlling and guiding traffic, guaranteeing traffic and personal safety, and reducing accidents and so on ${ }^{[1]}$. According to statistics of British investigations, the accident rate may declined by $75 \%$ within a section of highway where markings are already available ${ }^{[2]}$. As stipulated by China's “Quality Inspection and Evaluation Standards for Highway Engineering”, the roads that have been built shall meet corresponding criteria, among which marking is one of the important indices.

At present, highways are mainly marked by hand-propelled road marking machines and lowpressure air spray in China. Waterlines shall be placed on highways prior to marking due to lack of positioning devices. Under normal conditions, the waterlines are placed at a speed of $15 \mathrm{~km} / \mathrm{h}$, six people a vehicle. In countries like Britain and the United States have earlier developed specialized and highly automatic marking vehicles, where a sensing camera system is established, so new paints may be added by automatically tracking previous old lines with computer, thus increasing the coverage precision and work efficiency ${ }^{[3]}$. Nevertheless, except that they are automatic and selfdirected, foreign marking vehicles have no apparent advantages in marking technologies compared with hand-propelled road marking machines.. Currently, domestic research on marking vehicles focuses on exploration and development of spray systems, namely ambient-temperature highpressure air-free and hot-melt spray systems ${ }^{[4]}$. In developed foreign countries, there is relatively little work on road markings because roads are seldom built, so their research and development of road marking machines mostly focus on identification technologies for old markings ${ }^{[5]}$. There are rarely any studies concerning automatic positioning of marking vehicles home and abroad.

Due to high directivity and low energy attenuation, ultrasonic waves are propagated over a long distance through media and commonly used for ranging without high requirements for precision, such as ultrasonic range finders and level meters. Ultrasonic ranging system is widely used in general industries on the grounds of rapid/convenient detection, easy real-time control, low costs and conformance to general industrial requirements in terms of measuring precision. Current research aimed at developing a positioning system based on ultrasonic ranging for marking vehicles. During the movement of a marking vehicle, the ultrasonic ranging system is utilized to accurately determine relative position of reference objects relative to guardrails, for the purpose of offering technical support for accurate and efficient spray painting of road markings. 


\section{Ultrasonic Ranging Principle}

Ultrasonic ranging adopts "pulse-echo method", including integrated and separated receivingtransmitting ranging systems, among which the latter is shown in Fig 1. On the grounds that ultrasonic waves are propagated through homogenous media at a relatively stable speed that doesn't change with the frequency of sound waves, the distance may be measured by multiplying the time cost for receiving echo after transmitting ultrasonic wave by the velocity of sound.

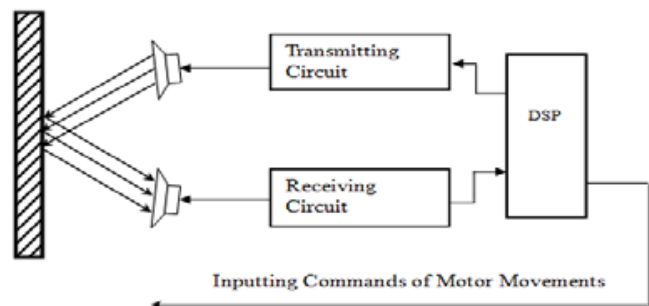

Fig.1 Sketch of UItrasonic Ranging Principle

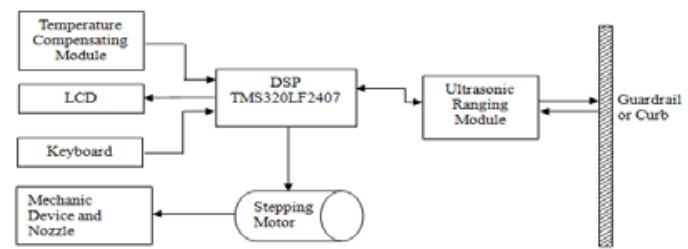

Fig.2 Structure of UItrasonic Ranging System

\section{Design of Ultrasonic Ranging System}

The overall structure of ultrasonic ranging system is shown in Fig 2 :

The system is composed of ultrasonic ranging module, DSP, temperature compensating module, LCD and keyboard. Ultrasonic waves are transmitted and data are acquired under the control of DSP which measure the position of a marking vehicle relative to highway guardrail and determine the deviation of the vehicle from predetermined marking track. Then, corresponding command is sent to order the stepping motor to exercise proper movements and drive marking nozzle to move to track the marking track. Besides, the temperature of working environment is measured with the temperature compensating module to compensate the velocity of sound, and the operating state of the system is displayed by LCD, while initial parameters are set by keyboard.

\subsection{Design of System Hardware}

\subsubsection{Ultrasonic Transmitting and Receiving Circuits}

The ultrasonic ranging module applies LM1812, a special chip for ultrasound, which is cheap with outstanding performances as a universal integrated device for transmitting and receiving ultrasonic waves. This chip is equipped with Class $\mathrm{C}$ oscillator for pulse modulation, high-gain receiver, pulse modulation detector and noise suppressor and so on inside.

\subsection{DSP}

This ranging system is mainly used for determining relative position of running vehicles and giving feedbacks on signals to control stepping motor and marking nozzle, so there are relatively high requirements for real-time properties. Therefore, TMS320LF2407 single-chip digital processor (supplied by a Texas instrument company) is used as control system. Designed for supporting highspeed control and applications with high requirements for digital operation, it is a reliable and cheap signal processor that integrates flexibility of a high-speed controller with digital processing abilities of an array processor.

\subsubsection{Temperature Compensating Circuit}

Temperature has certain impacts on precision of ultrasonic ranging. To satisfy precision-related requirements of the system, it is necessary to measure and compensate the temperature. Thus, the temperature was measured with the DS18B20 uniline digital temperature sensor manufactured by DALLAS, an American company of semiconductors. Besides, an interface circuit and a temperature compensating circuit of DS18B20 and DSP were designed for temperature compensation.

\subsubsection{LCD}

LCD is used for displaying information about the whole system, mainly including system operating state, distance to be measured, spraying amount and current temperature. Controlled by keyboard, it may still display set distance, measured distance and temperature and so on. On the grounds that only a small amount of information needed to be displayed, so a character-type LCD displaying 2 lines X 8 characters, Hitachi-driven HD44780U and 4/8-bit PPI were selected. 


\subsubsection{Power System and Reset Circuit}

At present, the power supply available from automobiles is mostly $12 \mathrm{~V}$. The ultrasonic ranging module applies a voltage of $12 \mathrm{~V}$ and can be directly used as lead for storage batteries. The voltage shall be $5 \mathrm{~V}$ in the peripheral circuit and 3.3V in DSP, so level conversion is required. In this system, the voltage of storage batteries is transformed by $78 \mathrm{~L} 05$ from $12 \mathrm{~V}$ to $5 \mathrm{~V}$ for the external circuit in an automobile. Then, it is converted by TPS76733 chip from 5V to 3.3V for DSP.

In addition, RS1, led by No. 8 lead of TPS76733 chip, was connected with RS of DSP. A watchdog reset circuit was added for the purpose of resetting.

2.2. Design of System Software

The software of DSP is primarily responsible for acquiring, processing, storing and controlling signals. The functional design of software mainly covers four modules as follows:

(1) System initialization module, used for setting initial parameter.

(2) Data acquisition module, utilized for transmitting and acquiring signals.

(3) Data processing module, for processing data about temperature and ultrasonic waves.

(4) Data output module, applied for controlling operating state of nozzle and correctly transmitting the data measured to the motor control module according to specific conditions.

The major task of software system is to initialize the system by setting the initial value of the distance between a marking vehicle and a reference object (guardrail) with keyboard. Next, the actual distance is calculated by the time difference between transmitting and receiving ultrasonic waves. Compared with the set distance, the value difference ranging from 0 to $1 \mathrm{~cm}$ will be considered as allowable range of error according to marking criteria. In this case, the system will be identified to work normally, open the nozzle and mark lines normally. Provided that the value difference is beyond $100 \mathrm{~cm}$, the error will be judged by the system to be caused by missing guardrail. Then, the nozzle will be suspended from working by the system, and normal spraying won't be recovered until the data on missing reference object is re-measured.

In case that the value difference ranges from $1 \mathrm{~cm}$ to $100 \mathrm{~cm}$, it means that the deviation results from running vehicles. At this moment, the system will modulate motor drive and output corresponding number of impulses based on the value of deviation, in order to control the movement of nozzle back to a correct position by the stepping motor. The normal spraying will be restarted until the deviation is recovered to the normal range. In consideration that temperature doesn't drastically change within a short period during system operation, the data on temperature is acquired every one to two hours by the system.

The flowchart of DSP Software is shown in Fig 3.

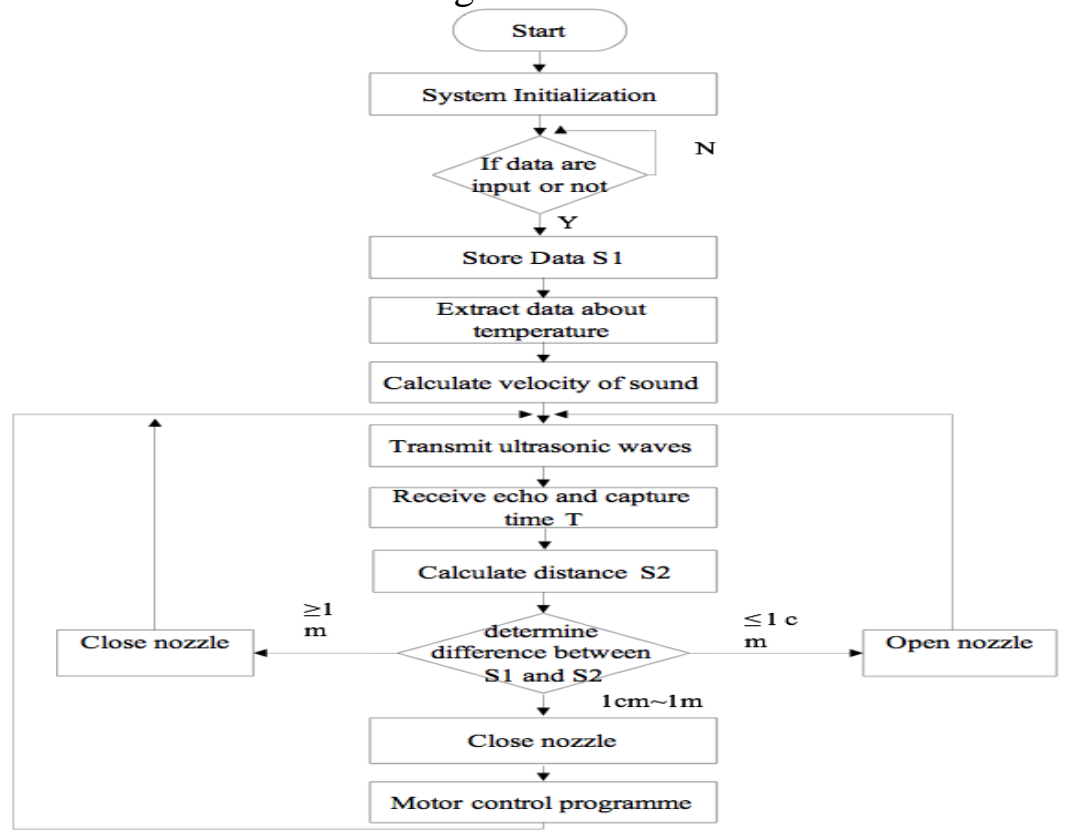

Fig 3. Flowchart of DSP Software 


\section{Simulation Test}

To determine measuring scope and precision of the system, a $850 \mathrm{~cm} \times 700 \mathrm{~cm}$ (height $\mathrm{X}$ width) wall was selected and the probe was erected $200 \mathrm{~cm}$ high and 7 distances were measured, including $100 \mathrm{~cm}, 200 \mathrm{~cm}, 300 \mathrm{~cm}, 400 \mathrm{~cm}, 500 \mathrm{~cm}, 600 \mathrm{~cm}$ and $700 \mathrm{~cm}$. The test results showed that at the point of more than $600 \mathrm{~cm}$, the calculated mean values were extremely close to actual values. As the distance to be measured gradually increased to $700 \mathrm{~cm}$, the system could arbitrarily receive normal signals and the measured data were generally correct. As a result, the measuring scope of an ordinary ultrasonic probe ranges from $0.6 \mathrm{~m}$ to $0.7 \mathrm{~m}$.

From the simulation test, it could be known that the measuring error of the ranging system was relatively high when the measuring distances is less than $100 \mathrm{~cm}$ and more than $500 \mathrm{~cm}$, and better effects in measuring medium-range distance is from 100 to $500 \mathrm{~cm}$.

\section{Conclusion}

(1) The ultrasonic ranging system based on DSP control has good real-time properties and may meet the needs for ranging and positioning with marking vehicles.

(2) This ultrasonic ranging system may effectively measure distance from 60 to $700 \mathrm{~cm}$ and it had less errors in the medium-range distance from 100 to $500 \mathrm{~cm}$.

\section{References}

[1] CLELAND B S, WALTON D, THOMAS J A. The relative effects of road markings on cycle stability[J]. Safety Science, 2005, 43:75-89.

[2] He Xinyun, Foreign Roads and Traffic Safety [J]. Traffic and Transportation, 1999 (1): 24

[3] Li Ke, Lin Jinmu. On Development of New Road Marking Machines [J]. Hunan Communication Science and Technology, 1995, 21 (4): 26-29.

[4] Wu Yunya, Kan Jiarong. Control System for Vibrating Road Marking Machine. Journal of Yancheng Institute of Technology (Natural Science Edition) [J], 2006, 19 (4): 31-33.

[5] Shen Songyun. Foreign Marking Machines [J]. Construction Machinery, 1998, 10: 38-39. 